\title{
Umwege oder: Versuch einer Einführung in Leben und Werk Heimito von Doderers
}

\section{Detours, or an Attempt at an Introduction to the Life and Work of Heimito von Doderer}

\author{
Manfred Müller
}

\begin{abstract}
Umwege, or detours, are a valid and valuable motif to follow in examining the life and work of Heimito von Doderer, as has also been noted by others, most prominently by Wendelin Schmidt-Dengler. Doderer himself uses the term frequently, even as the title of an early novel. This essay presents evidence to demonstrate that virtually nowhere in Doderer's life did anything follow a straight line, be it his career, his philosophy of life, his political orientation, or his religious persuasion. Similarly, his literary output, most particularly Die Strudlhofstiege and Die Dämonen, meanders from the conventional form of the modern novel, following an intricate orchestral-style score rather than a straightforward plot. In this sense, it is unsurprising that Doderer chose the Strudlhofstiege with its serpentine system of ramps and platforms as his iconic visual focus in Vienna's $9^{\text {th }}$ District, the main setting of Die Strudlhofstiege.
\end{abstract}

\section{Keywords}

Heimito von Doderer; detour; poetics; philosophy of life

Dieser Beitrag ist die schriftliche Fassung eines allgemeinen, in Doderers Leben und Werk einführenden Vortrags, der am 5. Dezember 2017 vor Studierenden der Masaryk-Universität in Brno gehalten wurde. 
Es ist keine neue Idee, sich dem Autor Heimito von Doderer und seinem Werk über das Motiv des Umwegs zu nähern. Unter anderen (aber vor allem) hat schon Wendelin Schmidt-Dengler, einer der wichtigsten Doderer-Forscher überhaupt, eine Einführung mit einem Exkurs über den Umweg begonnen. ${ }^{1}$ Der Grund dafür liegt auf der Hand: Der Begriff kommt bei Doderer häufig vor. Zum einen spielt er eine zentrale Rolle für diverse Theorien und Überlegungen, die grundlegend für seine schriftstellerische Praxis ebenso wie für seine Lebensphilosophie wurden. Ein Umweg ist zudem der Titel eines 1940 von ihm veröffentlichten Romans. Zum anderen drängt sich der Begriff auch beim Blick von außen - egal ob auf Doderers Leben oder sein Werk - in mehrfacher Hinsicht geradezu auf, beispielsweise wenn man, wie auch Schmidt-Dengler das getan hat, mit seinem sicherlich berühmtesten Roman und dessen titelgebendem Objekt, der Strudlhofstiege im 9. Wiener Gemeindebezirk, beginnt. Das hat den einfachen Grund, dass dieses Bauwerk einem Umweg in geradezu idealer Weise entspricht (und von Doderer wohl auch deshalb ausgewählt wurde). Der vergleichsweise geringe Höhenunterschied wird mittels mehrerer serpentinenartig angelegter Rampen überbrückt. Als Fußgänger legt man daher einen ungewöhnlich langen Weg zurück und benötigt dafür wesentlich mehr Zeit, als dies bei einer einläufig ohne Umweg gerade ansteigenden, dafür aber steileren Stiege der Fall wäre. Schmidt-Dengler schreibt dazu: „Das Monument der Strudlhofstiege ist somit das beste Sinnbild für eine Umwegigkeit, die [nach Doderer (d. Verf.)] jede künstlerische Arbeit auszuzeichnen hätte, es ist das romanexterne Korrelat zur ästhetischen Konzeption des Werks und zugleich Versinnlichung eines Arbeitsprozesses, der jede direkte Aussage verschmäht und nicht einmal als zweitrangig gelten läßt." ${ }^{\text {2 }}$

Den Begriff des Umwegs als einen möglichen Weg zu Doderer zu definieren, ist natürlich auch eine Vereinfachung; zu monumental ist sein Werk, um es in der notwendigen Kürze auch nur ansatzweise vorstellen zu können. Ich werde mich daher zwangsläufig auf einige Aspekte beschränken, auf solche vor allem, die mir wichtig erscheinen, die meine Lektüre und Re-Lektüre zu einem Vergnügen gemacht haben und machen. Immerhin steht bei einem Autor, dessen Bücher allein durch ihren Umfang selbst im universitären Bereich bedeutende Lesehürden darstellen, ja unweigerlich auch die Frage ,Warum soll man Doderer lesen?‘ im Raum. Die Beispiele und Themenbereiche, die ich in der Folge kurz ansprechen möchte, stellen daher auch den Versuch einer Antwort auf diese Frage dar. Doderer erscheint hier nicht nur als Klassiker und Verfasser umfangreicher Romane, sondern auch als die möglicherweise ein wenig abwegige Figur, die er war und die in allen seinen Werken auf spannende Art zutage tritt.

1 Vgl. Schmidt-Dengler, Wendelin: Heimito von Doderer 1896-1966. In: ders: Jederzeit besuchsfähig. Über Heimito von Doderer. Hrsg. v. Gerald Sommer. München 2012, S. 249-270, hier: S. 249 f.

2 Schmidt-Dengler (Anm. 1), S. 250. 
Schon in der Biografie des Autors verstecken sich zahlreiche Umwege, die Doderer auch selbst des Öfteren thematisiert hat. Als ersten und längsten davon könnte man den letztlich mehrere Jahrzehnte dauernden Umweg aus der Familie heraus bezeichnen: Heimito von Doderer wurde 1896 in eine der wohlhabendsten Familien der Doppelmonarchie hineingeboren. Der nahezu lebenslange Versuch, sich von seiner Familie zu distanzieren, vor allem aber von seinem despotischen Vater, der typisch großbürgerliche Erwartungen an den offensichtlich wenig geschäftstüchtigen Sohn stellte, gelang im Grunde erst Jahrzehnte nach dessen Tod mit dem späten Erfolg als Schriftsteller, den Die Strudlhofstiege und Die Dämonen mit sich brachten, und ist von verschiedenen kleineren biografischen Umwegen geprägt bzw. begleitet worden. Einige davon möchte ich hier nennen:

1) Der Umweg zum Schriftsteller ergab sich für Doderer während der in russischer Kriegsgefangenschaft verbrachten Jahre infolge seiner Teilnahme am Ersten Weltkrieg. Im Juli 1916 bei Olesza in Ostgalizien in Gefangenschaft geraten, lernte er in sibirischen Offizierslagern eine Reihe junger Künstler und Intellektueller kennen - Begegnungen, die seinen Weg nachhaltig prägen und seine ersten Verlagskontakte und Publikationen ermöglichen sollten.

2) Nach seiner Rückkehr nach Wien im Jahr 1920 studierte er aber zunächst Geschichte und Psychologie und begann 1927, neben literarischen auch eine Vielzahl journalistischer Texte zu veröffentlichen. Erst mit der Einsicht, dass das Schreiben für Zeitungen und Magazine seinen künstlerischen Zielen im Wege stand, begann er sich ab 1930 immer mehr auf literarische Arbeiten zu konzentrieren, worin ihn vor allem der Schriftsteller und Maler Albert Paris Gütersloh - sein künftiger „verehrter Meister“ - bestärkte.

3) Ein weiterer, in vielen biografischen Abrissen häufig thematisierter Umweg, nämlich jener in die NSDAP (sein Beitritt erfolgte am 1. April 1933), führte groteskerweise über die 1930 geschlossene Ehe mit Gusti Hasterlik, der Tochter eines Zahnarztes jüdischer Herkunft, mit der er bereits seit 1920 eine Liebesbeziehung hatte.

4) Über den Nationalsozialismus erfolgte dann ein weiterer ungewöhnlicher Umweg: Der protestantisch getaufte und zeitweilig konfessionslose Doderer konvertierte 1940 zum Katholizismus, der fortan große Bedeutung für ihn und sein Schaffen hatte.

5) Über all diese (und auch trotz solcher) Bocksprünge, nach neuerlichem Wehrdienst im Zweiten Weltkrieg und dem Hunger der unmittelbaren Nachkriegszeit, wurde er schließlich in den 1950er-Jahren zu dem gefeierten Staatsdichter der Zweiten Republik.

Man könnte Doderers Umwege auch in vielen anderen Bereichen suchen und finden. Parallel zur Biografie ist nämlich auch sein Werk durch Umwege geprägt. Dies beginnt schon mit der Wahrnehmung durch das Publikum: Die Liste von Doderers Veröffentlichungen ist lang, doch erschien ein großer Teil davon nahezu unter Ausschluss der Öffentlichkeit. Der Durchbruch gelang Doderer erst 1951 mit seinem sechsten Roman im Alter von 54 Jahren. Sein Erfolg währte daher auch nur etwas mehr als ein Jahrzehnt, ehe er während der Arbeit an seinem vierteilig angelegten Roman No 7 am 23. Dezember 1966 an Darmkrebs starb. 
Poetologisch führt ein markanter Umweg von frühen, expressionistisch geprägten Texten über die Verinnerlichung der Theorien Albert Paris Güterslohs zum eigenständigen, das Spätwerk prägenden Projekt des „roman muet“, in dem der Autor völlig in den Hintergrund treten sollte.

Und auch der eigentliche poetische Akt ist von Umwegen geprägt: Doderer verfasste seine Texte nicht in einem Arbeitsschritt. In mehreren Schreibphasen, mit viel Disziplin und großem Aufwand, fanden seine Arbeiten über Skizzen- und Tagebücher sowie Manuskriptbände in sorgsam, auch vom Schriftbild her klar getrennten Schritten zur endgültigen Form.

Schließlich lässt sich ein historisch-philosophischer Umweg in Doderers Denken konstruieren: Seine Annäherung an die Gegenwart, an die Bewältigung der eigenen Existenz angesichts politischer Irrungen und Vereinnahmungen erfolgte über lange historische Umwege: Mit der Lektüre von Thomas von Aquins De ente et essentia fand er zur Weltsicht der mittelalterlichen Scholastik und damit auch zu einer - seiner Meinung nach - zeitgemäßen Bewältigung der Gegenwart, parallel dazu, über Sprache, Zucht und Ausschweifungen des Mittelalters zu seiner eigenen (Ausdrucks-)Form sowie zum Verständnis und zu für ihn gültigen Erklärungen vieler seiner Überzeugungen und Vorlieben, aber auch Probleme.

Zusammenfassend erscheint vor allem der politische Umweg spektakulär: vom Großbürgersohn mit aristokratischem Gehabe, der eine Zeitlang mit der bolschewistischen Revolution sympathisierte, sich seit Anfang der 1930er-Jahre dem Nationalsozialismus annäherte, aber - ab August 1936 im Deutschen Reich lebend - bereits 1937 wieder begann, auf Distanz zu diesem zu gehen, zum konservativen Repräsentanten der Zweiten Österreichischen Republik. Doderer selbst hat in seinen späten Jahren zum einen die Kontinuität in seiner Gesinnung über die Jahre hinweg betont, zum anderen aber doch auch, wenngleich nicht ganz leicht erkennbar, das Problematische an seinen politischen Bocksprüngen thematisiert. So handelt der erste große Essay, den er nach dem Zweiten Weltkrieg schrieb, von Schuld, wenngleich in ihrer Umkehrung: „Von der Unschuld im Indirekten“ ist der Titel dieses unter dem Pseudonym René Stangeler, dem Namen einer in der Folge wichtigen Romanfigur in Die Strudlhofstiege und Die Dämonen, veröffentlichten Texts, und Doderer beschreibt darin vordergründig seinen älteren Autorenkollegen und Freund Albert Paris Gütersloh; tatsächlich aber geht es darin auch um sein eigenes Dasein als unpolitischer Schriftsteller, als sich mit geistigen Dingen Beschäftigender auf der Suche nach der Sprache. Letztlich dient „Von der Unschuld im Indirekten“ der Etablierung einer neuen Begriffsebene:

Direkt bedeutet vollkommen gerade; das kommt im Leben, welches nur Kurven kennt, nicht vor; es bedeutet aber auch (als participium von dirigo) „gelenkt“: und das heißt, in den psychologischen Jargon übersetzt, „bewußt“.

Durch bewußtes Denken, das seinen Gegenstand genau dem Subjekte gegenüber in den Schraubstock spannt und im Kerne des Lichtkegels hält, gelangt niemand zur Sprache. ${ }^{3}$

3 Doderer, Heimito von: Von der Unschuld im Indirekten. Zum 60. Geburtstag Albert P. Güterslohs. In: 
Das Indirekte, das man auch synonym mit Doderers Theorien vom Umweg verwenden könnte, führt demnach letztlich zur Sprache. Indirekt meint sehr vieles: unbewusst, ungelenkt, absichtslos und manches andere mehr. „Von der Unschuld im Indirekten“ ist aber auch ein Eingeständnis, sich dem Leben, genauer: dem Politischen, zu direkt, zu unmittelbar ausgesetzt zu haben. Im Direkten steckt für Doderer die Schuld, und dass damit auch die eigene, also die Verstrickung in nationalsozialistisches Gedankengut, gemeint sein könnte, zeigen einige wenige Texte, vor allem die späte Erzählung „Unter schwarzen Sternen“. Dazu passen auch Aussagen wie die folgende, die einen ganz besonderen, persönlichen Umweg beschreibt: „Mein eigentliches Werk besteht, allen Ernstes, nicht aus Prosa oder Vers: sondern in der Erkenntnis meiner Dummheit."

\section{3}

Aber kommen wir zum Erzählen, zu den großen Romanen: Sie sind teils außerordentlich umfangreich, sie widersetzen sich nicht selten unseren Lesegewohnheiten, und doch schaffen sie es, uns zu fesseln.

Doderer lässt sich schwer mit anderen vergleichen, schon gar nicht mit den groBen Epikern unter seinen Zeitgenossen, von Robert Musil über Thomas Mann und Hermann Broch bis hin zu Alfred Döblin oder James Joyce. Was das Besondere an seinem Schreiben ausmacht, ist zunächst nicht einfach zu erklären.

Ein Satz aus Die Strudlhofstiege erscheint mir wie ein kleiner Wegweiser, ein Fingerzeig auf das, was hinter der Komplexität von Doderers großen Romanen stecken könnte: „Wir hinterlassen unsere Spuren aneinander. “5 Er ist auf zwei Figuren bezogen, deren Verhältnis zu- und Kenntnis voneinander nach Jahren gewisse Eigenheiten des einen beim jeweils anderen erklärt: Im konkreten Fall ist eine Gewohnheit der Figur E.P. die Ursache dafür, dass sein ehemaliger Freund, der bereits angesprochene René von Stangeler, keine Hosenträger mehr verwendet.

Was hier banal erscheint, lässt sich, allgemeiner betrachtet, auf die Konstruktion der großen Romane Doderers und sein Spätwerk anwenden: In diesen Texten mit ihrem gewaltigen Figurenarsenal gibt es niemand, der autonom für sich allein agieren würde. Alles, was gemacht, gedacht, versucht wird, hängt unmittelbar von Ursachen ab, die wiederum mit anderen Figuren zusammenhängen. Sehr rasch entstehen auf diese Weise Netze, die sich durch das ganze Buch ziehen. Alles, jede und jeder hängen mit allem, jeder und jedem zusammen. Doderers Spiel der Verknüpfungen wird mit der Länge der

ders.: Die Wiederkehr der Drachen. Aufsätze / Traktate / Reden. Hrsg. v. Wendelin Schmidt-Dengler. Mit einem Vorwort v. Wolfgang H. Fleischer. München 1970, S. 111-125, hier: S. 117.

4 Doderer, Heimito von: Meine neunzehn Lebensläufe. In: ders.: Meine neunzehn Lebensläufe und neun andere Geschichten. Zum 70. Geburtstag des Autors. Mit 19 Fotos und einer Schallplatte. München 1966, S. 7-36, hier: S. 28.

5 Doderer, Heimito von: Die Strudlhofstiege oder Melzer und die Tiefe der Jahre. München 1951, S. 24. Vgl. a. Sommer, Gerald: Heimito von Doderer: „Technische Mittel“. Fragmente einer Poetik des Schreibhandwerks. Mit e. Vorwort d. Herausgebers [Wendelin Schmidt-Dengler]. Wien 2006 (Zur neueren Literatur Österreichs; 21), S. $8 \mathrm{f}$. 
Romane logischerweise immer unübersichtlicher, und der Erzähler, obwohl oft scheinbar geschwätzig erklärend und kommentierend, gibt selbst vor, nicht alle Zusammenhänge zu verstehen. Darum geht es aber auch gar nicht: Diese Komplexität der Welt ist es gerade, die Doderer darstellen möchte. Und als solche ist sie eben vom Einzelnen kaum $\mathrm{zu}$ beeinflussen, keinesfalls aber mehr als ansatzweise zu verstehen.

Ich möchte versuchen, das mit einem Vergleich aus einem ganz anderen Feld zu erklären: Beim Billardspiel versucht man, vorauszusehen, wohin die gestoßene Kugel geht, auch nachdem sie eine andere oder die Bande berührt hat. Professionelle Spieler schaffen es, mehrere solcher Kollisionen, die daraus resultierenden Richtungsänderungen der Kugeln sowie deren daraus erfolgende Lage auf dem Tisch recht genau vorherzusagen. Ab einer gewissen Anzahl an Kontakten ist eine Vorhersage aber unmöglich.

Natürlich sind Doderers monumentale Romane wesentlich komplexer als vergleichsweise kleine Billardtische, die mit drei, 16 oder (beim Snooker) 22 Kugeln bespielt werden: Allein im Kosmos der beiden Romane Die Strudlhofstiege und Die Dämonen, die letztlich auch als ein einziger großer Roman gelesen werden könnten, treten weit mehr als 300 Figuren auf. Nahezu jede davon trifft viele andere, fast alle haben ganz bestimmte Vorhaben, Ziele, Wünsche, die natürlich allesamt immer die Vorhaben, Ziele und Wünsche anderer beeinflussen. Ich denke, das erklärt schon, wie schwierig es ist, klassische, nacherzählbare Romanhandlungen bei Doderer zu finden - und eines seiner berühmtesten Zitate ist daher nicht ohne Grund ein Wort aus dem Jahr 1966: „Ein Werk der Erzählungskunst ist es um so mehr, je weniger man durch eine Inhaltsangabe davon eine Vorstellung geben kann." ${ }^{\circ}$

Kombiniert man die beiden zuletzt formulierten Hypothesen, nämlich erstens, Doderers Ziel sei es, die Welt in all ihrer Komplexität und Unübersichtlichkeit darzustellen, und zweitens, der Inhalt als nacherzählbarer roter Faden, der eine Geschichte üblicherweise zusammenhält, sei bei Doderer vergleichsweise unwichtig, dann ist man, denke ich, auf einem guten Weg.

Doderer selbst hat mit dem, was daraus folgen könnte, gespielt, unter anderem in einer kurzen Erzählung mit dem Titel „Die Mitteilung“:

„Wann ist denen die Sache mitgeteilt worden?“ - „Gegen halb zwölf.“ - „Haben die was gesagt, haben sie eine Wut gekriegt?“ - „Nein. Kein Wort haben sie gesagt, haben sich überhaupt nicht gerührt. Keinerlei Äußerung wurde getan.“ - „Und weiter? Und sonst nichts?“ - „Doch. Gegen sechs Uhr abends sind sie alle zugleich aus ihrem Bau gefahren und haben die Leute entsetzlich verprügelt, ja, geradezu gedroschen. Alles ist gelaufen, die Leut' haben nur geschaut, daß sie weiterkommen, um nicht auch noch erwischt und verdroschen zu werden ..." „Ja, was für Leute haben denn die verhauen?“ - „Nicht jemand bestimmten. Wer halt grad vorbeigekommen ist. Einen kleinen alten Mann haben sie derart getreten, daß er mit dem

6 Doderer, Heimito von: Repertorium. Ein Begreifbuch von höheren und niederen Lebens-Sachen. Hrsg. v. Dietrich Weber. München 1969, S. 72. 
Gesicht aufs Kanalgitter gefallen ist. Es war fürchterlich.“ - „Und was ist denen eigentlich um halb zwölf mitgeteilt worden?" - „Das weiß ich nicht."7

Hier passiert doch einiges, eigentlich sogar recht viel, es kommen zahlreiche Personen vor, sogar die Zeit ist auf eine halbe Stunde genau angegeben. Je mehr man aber wissen will, desto weniger wird einem letztlich klar. Weder die Fragen nach dem Wer noch nach dem Wie oder Wo werden beantwortet - vor allem aber fehlt die Antwort auf das Warum. Interpreten, die es gewohnt sind, Texte zu analysieren, nach Motivierungen aller denkbaren Handlungen in literarischen Texten zu suchen, beißen sich, so scheint es, an diesem von Doderer so genannten „Wutanfall“ die Zähne aus.

Genau dies ist, denke ich, das Wichtige: Doderer gibt oft weder einen genauen Weg vor noch gibt er Rätsel auf, die lösbar wären. Sein Anspruch ist es, die Komplexität und Unerklärbarkeit - der Welt zu belassen und abzubilden.

\section{4}

Dass Doderers Werke trotzdem funktionieren, man sie also gerne liest, sich darin zurechtfindet (und nicht einfach veralbert vorkommt), liegt daran, dass der Autor diese Komplexität letztlich an Einzelheiten aufhängt und damit Bezugspunkte herstellt, deren Entwicklungslinien man folgen kann. Und natürlich sieht er es als seine wichtigste Aufgabe an, die Zügel nicht aus der Hand zu geben: Er behält den Überblick, stellt und bewegt sein Romanpersonal wie Figuren auf einem Schachbrett. Und tatsächlich hat er seine Romane, vor allem aber das Figurenarsenal und wie es zusammenhängt, auf großen Reißbrettern zeichnerisch festgehalten, um den Überblick zu behalten.

In Doderers Erzählwerk steckt daher, wenn man so will, ein Paradoxon: Die Offenheit einer möglichst realitätsgetreuen Weltdarstellung wird dadurch ermöglicht, dass der Autor mit größtmöglicher Präzision und absoluter Kontrolle vorgeht. Der Rolle des Autors als Kontrollinstanz entspricht, dass Doderer dabei ein Konzept im Sinn hatte, das er - ausgehend von Gütersloh - entwickelt hatte: das des sogenannten „totalen Romans“. Dieses Konzept beeinflusste vor allem die beiden großen Romane Die Strudlhofstiege und Die Dämonen. Daher lassen sie sich kaum in gängige literaturhistorische Kategorien einordnen; sie sind vielmehr komplexe Geflechte aus verschiedenartigsten Handlungssträngen, Kombinationen aus Entwicklungs- und Familienromanen, aus Zeit- und Gesellschaftsromanen, aus Liebes- und Intrigenromanen und anderem mehr.

Worum es aber immer geht, ist nicht, eine Geschichte zu erzählen, sondern mithilfe vieler Geschichten und auch, im wienerischen Sinn, mit Gschichteln, die Totalität der Welt mit all ihren Unregelmäßigkeiten und Gesetzmäßigkeiten abzubilden. Über das Lesen von Doderers Texten lässt sich zudem eines mit Sicherheit sagen: dass man sich permanent auf Umwegen befindet, durchaus auch auf Irrwegen. Man folgt den Fährten, die der Autor legt, um letztlich ganz woanders anzukommen, an Punkten, die man nicht

7 Doderer, Heimito von: Die Mitteilung. In: ders.: Die Erzählungen. Hrsg. v. Wendelin Schmidt-Dengler. München 1972, S. 310. 
vorhersehen hätte können. Diese Umwege des Lesens, die einem Doderer - und sicher hat er dabei großen Genuss und auch ein wenig Schadenfreude verspürt - aufoktroyiert, machen das Lesen seiner Bücher zu einem besonderen Vergnügen.

\section{Literaturverzeichnis}

DODERER, Heimito von (1951): Die Strudlhofstiege oder Melzer und die Tiefe der Jahre. München.

DODERER, Heimito von (1966): Meine neunzehn Lebensläufe. In: ders.: Meine neunzehn Lebensläufe und neun andere Geschichten. Zum 70. Geburtstag des Autors. Mit 19 Fotos und einer Schallplatte. München, S. 7-36.

DODERER, Heimito von (1969): Repertorium. Ein Begreifbuch von höheren und niederen Lebens-Sachen. Hrsg. v. Dietrich Weber. München.

DODERER, Heimito von (1970): Von der Unschuld im Indirekten. Zum 60. Geburtstag Albert P. Güterslohs. In: ders.: Die Wiederkehr der Drachen. Aufsätze / Traktate / Reden. Hrsg. v. Wendelin Schmidt-Dengler. Mit einem Vorwort v. Wolfgang H. Fleischer. München, S. 111-125.

DODERER, Heimito von (1972): Die Mitteilung. In: ders.: Die Erzählungen. Hrsg. v. Wendelin Schmidt-Dengler. München, S. 310.

SCHMIDT-DENGLER, Wendelin (2012): Heimito von Doderer 1896-1966. In: ders: Jederzeit besuchsfähig. Über Heimito von Doderer. Hrsg. v. Gerald Sommer. München, S. 249-270.

SOMMER, Gerald (2006): Heimito von Doderer: „Technische Mittel“. Fragmente einer Poetik des Schreibhandwerks. Mit e. Vorwort d. Herausgebers [Wendelin Schmidt-Dengler]. Wien (Zur neueren Literatur Österreichs; 21).

Dr. Manfred Müller / office@ogl.at

Österreichische Gesellschaft für Literatur

Herrengasse 5

A-1010 Wien, AT 